\title{
Construction of Sparse Representations of Perfect Polyphase Sequences in Zak Space with Applications to Radar and Communications
}

\author{
Andrzej K. Brodzik \\ The MITRE Corporation, Emerging Technologies, Bedford, MA 01730, USA \\ Correspondence should be addressed to Andrzej K. Brodzik, abrodzik@mitre.org \\ Received 1 July 2010; Accepted 9 September 2010 \\ Academic Editor: Antonio Napolitano \\ Copyright ( 2011 Andrzej K. Brodzik. This is an open access article distributed under the Creative Commons Attribution License, \\ which permits unrestricted use, distribution, and reproduction in any medium, provided the original work is properly cited.

\begin{abstract}
Sparse representations of sequences facilitate signal processing tasks in many radar, sonar, communications, and information hiding applications. Previously, conditions for the construction of a compactly supported finite Zak transform of the linear FM chirp were investigated. It was shown that the discrete Fourier transform of a chirp is, essentially, a chirp, with support similar to the support of the time-domain signal. In contrast, the Zak space analysis produces a highly compactified chirp, with support restricted to an algebraic line. Further investigation leads to relaxation of the original restriction to chirps, permitting construction of a wide range of polyphase sequence families with ideal correlation properties. This paper contains an elementary introduction to the Zak transform methods, a survey of recent results in Zak space sequence design and analysis, and a discussion of the main open problems in this area.
\end{abstract}

\section{Introduction}

In this paper, we are concerned with the design and analysis of perfect polyphase sequences. A complex-valued sequence is polyphase when it has constant magnitude. A sequence is perfect when it has ideal correlation properties; that is, when it has zero out-of-phase autocorrelation and minimum cross-correlation sidelobes. A complex-valued sequence is a perfect polyphase sequence (PPS) when it is both polyphase and perfect. The design of PPSs has a long history, with deep connections to several branches of mathematics and engineering. While it is not possible to give a full account of this history here, we will remark on a few landmark developments. For a more extensive treatment of this subject the reader is referred to [1-3].

Some of the fundamental mathematical ideas underlying sequence design can be traced back to the work of Gauss on the quadratic reciprocity law [4] and to the works of Sidon [5], Erdös [6], and Littlewood [7] on certain polynomials with integer coefficients. One common theme in these otherwise rather diverse works is the focus on sequences that contain, in a certain sense, the least amount of redundancy $[8,9]$. However, these works have not been noted for their relevance to engineering applications until much later, when sequence design became a well-established subdiscipline of both radar and communications. This process started some fifty years ago, after the publication in 1953 of Woodward's book Probability and Information Theory with Applications to Radar [10], which for the first time brought attention to sequence design as an engineering problem. Subsequently, many important results have been obtained. In 1960, Klauder et al. published the seminal paper "The theory and design of chirp radar" [11]. In the next several decades, the utility of a family of PPSs in communication systems was recognized and many new families were proposed: see, for example, $[5,8,12-19]$. With the recent advances in digital electronic systems, these families could be realized in hardware and used in advanced signal processing tasks, such as the design of multibeam radar waveform sets for complex scene interrogation [20], multiple-user interference rejection [21], low probability of detection schemes [22], and spread spectrum multiple access communications, watermarking, 
and cryptographic systems [1, 23]. These efforts resulted, among others, in improved SNR, better clutter rejection, more efficient bandwidth allocation, and the design of new schemes for hiding information. The work in these areas continues, and many new discoveries are being made in both theoretical and applied domains. However, it is a testimony to the difficulty of this field that despite great many efforts undertaken over the last fifty years, the basic question of how to design a PPS remains substantially unanswered [24].

In this paper, we attempt to address the sequence design problem in Zak space. This choice requires an elucidation. One of the key properties of a PPS is that its discrete Fourier transform (DFT) is also polyphase $[2,25]$. This means that both a PPS and its DFT are nonzero everywhere. This property is sometimes referred to as biunimodularity [12]. While biunimodularity can be desirable, since it facilitates, among others, the design of a low-power and largebandwidth radar [26], it makes the analysis of signals difficult [27]. To address this problem, it is useful to consider PPSs on a time-frequency plane. By back-projecting the intrinsically high-dimensional PPS onto an analysis space of a matching dimension, such as a time-frequency space, one can obtain a sequence representation that is highly localized in that space. This localization facilitates many sequence analysis tasks, including parameter estimation, noise and interference rejection, and detection. As an additional benefit, transferring the analysis to a higher dimensional space avails a host of geometric techniques that are often more effective and/or efficient than one-dimensional algebraic computations.

The general idea of casting sequence design in a timefrequency setting is not new. The analysis of the canonical PPS - the LFM chirp-in the intermediate spaces was first suggested over forty years ago in the context of radar by Lerner [28]. Since then, many time-frequency settings for chirp signal processing have been proposed [29]. The best known examples include frameworks based on the Wigner distribution [30], the spectrogram [31], wavelets [32], and the fractional Fourier transform [33, 34]. Here, we describe an alternative approach based on the finite Zak transform (FZT). The finite Zak space approach is advantageous for several reasons. First, the Zak transform is closely related to the Fourier transform, and, therefore, Zak space analysis is a natural extension of Fourier space analysis. Second, since the Zak transform is linear, there are no cross-terms that occur in the quadratic time-frequency representations, such as the Wigner distribution. Third, the Zak transform does not require the use of a "window" signal, which often increases complexity and sometimes impacts the stability of the computation [35].

In prior work, we explored the utility of the Zak transform for PPS design focusing initially on the finite LFM chirp. It was shown that the DFT of a finite chirp is, essentially, a finite chirp, with support identical to the support of the time-domain sequence [36]. In contrast, the Zak transform produces a compact chirp image, with support restricted to an algebraic line [27]. Further research led to the relaxation of the original restriction to chirps, permitting the design of more general polyphase signals with ideal correlation properties [25]. The main results of the investigation, closely associated with these findings, include:

(1) closed-form expressions for the DFT and the FZT of the linear FM chirp, parameterized by chirp rate, carrier frequency and signal length,

(2) construction of Zak space sparsity conditions for the linear FM chirp and rules for chirp parameter estimation and chirp waveform recovery,

(3) design of large collections of new waveform sets with good auto and cross-correlation properties that include finite chirps, Zadoff-Chu sequences, and generalized Frank sequences as special cases,

(4) a new time-frequency space framework for the construction of PPS sets,

(5) a new time-frequency space framework for the analysis of chirps and chirp-like radar waveforms.

The last three results rely, in part, on the discovery that the Zak space representation of a PPS can be expressed as a composition of modulation and permutation operators acting on the canonical chirp sequence. This is an important result. Apart from aiding the design of "ordinary" PPSs, decoupling modulation and permutation can also be used for other purposes, such as the design of almost perfect sequences and perfect sequences with additional special properties.

Part of this work has been described in the Springer book [37] and in IEEE journals [25, 27, 36]. These presentations were written at a relatively advanced level in that they used concepts from both number theory and group theory to derive certain key results. The discussion in this paper is both broader in scope and more elementary. Following a brief introduction to the Zak transform calculus, we discuss the special relationship of the FZT with the DFT, the geometric character of the Zak space correlation, the Zak space implementation of the matched filter, the construction of the canonical PPS family, the perfect chirp set (PCS), and the generalizations of the PCS model. We conclude with a review of the main open problems in Zak space PPS design.

An unusual feature of this presentation is the joint focus on radar and communication applications. We will show that time-frequency analysis of a single classical radar waveform-the LFM chirp-leads to more general results that are relevant to all polyphase sequences. While many of these sequences are traditionally associated with communications applications, they can also be used in radar. Similarly, the sparse and highly structured support of PPS waveforms in the time-frequency space can be used advantageously in both radar and communications applications. These findings demonstrate that even though historically sequence/waveform design in the two fields progressed largely independently, the theoretical underpinnings are essentially the same, and hence a great deal of insight can be gained from juxtaposing ideas and results. 


\section{The Finite Zak Transform}

Zak was the first to make a systematic study of the transform that bears his name [38]. The Zak transform has several applications in mathematics, quantum mechanics, and signal analysis $[35,39,40]$. Here, we will state, without proofs, the properties of the FZT that are relevant to our constructions. For a more extensive review of Zak transform theory the reader is referred to [41] and a chapter in [42].

The FZT can be thought of as a generalization of the DFT. Therefore, a convenient way to describe it is to compare its basic properties with the properties of the DFT.

Take $x$ to be any $N$-periodic sequence in $\mathbb{C}^{N}$ and set $e_{L}(j):=e^{2 \pi i j / L}$. The DFT of $x$ is

$$
\mathbf{x}(m)=\sum_{n=0}^{N-1} x(n) e_{N}(n m), \quad 0 \leq m<N .
$$

Suppose that $N=K L^{2}$, where $L$ and $K L$ are positive integers. Then, the FZT of $x$ is given by

$$
X_{L}(j, k)=\sum_{r=0}^{L-1} x(k+r K L) e_{L}(r j), \quad 0 \leq j<L, 0 \leq k<K L .
$$

It follows from (2) that computing $X_{L}(j, k)$ requires $K L L$ point DFTs of the data sets

$$
x(k), x(k+K L), \ldots, x(k+(L-1) K L), \quad 0 \leq k<K L .
$$

Like the DFT, the FZT is a one-to-one mapping. A signal $x$ can be recovered from its FZT by

$$
\begin{array}{r}
x(k+r K L)=L^{-1} \sum_{j=0}^{L-1} X_{L}(j, k) e_{L}(-r j), \\
0 \leq r<L, \quad 0 \leq k<K L .
\end{array}
$$

Among the most fundamental properties of the FZT are shifts. Take $0 \leq j<L$ and $0 \leq k<K L$ for the remainder of this paper, unless indicated otherwise. The FZT is periodic in the frequency variable and quasiperiodic in the time variable, that is,

$$
X_{L}(j+L, k)=X_{L}(j, k) \text {, }
$$

and

$$
X_{L}(j, k+K L)=X_{L}(j, k) e_{L}(-j) .
$$

A related property describes the FZT of time and frequency shifts of $x$. Set $y(n)=x(n-c)$ and $z(n)=x(n) e_{N}(d n)$, where $0 \leq c<K L$ and $0 \leq d<L$. Then, the FZTs of $y$ and $z$ are given by

$$
Y_{L}(j, k)=X_{L}(j, k-c),
$$

and

$$
Z_{L}(j, k)=X_{L}(j+d, k) e_{N}(d k) .
$$

The properties (5)-(8) follow directly from (2). The relationship of the FZT to the DFT and the Zak space crosscorrelation formula are discussed separately in Sections 4 and 5 , respectively.

\section{The Linear FM Chirp}

One of the most ubiquitous waveforms in radar is the linear FM chirp. In this section, we state several key results on chirps, including the chirp discretization, the finite support condition, and the FZT formula. These results will be used later to construct more general sequences with optimal correlation properties. by

Define the linear FM signal, or the continuous chirp [11],

$$
x(t)=e^{\pi i \alpha t^{2}} e^{2 \pi i \beta t}, \quad \alpha \neq 0,0 \leq t<T,
$$

where $T$ is the chirp time duration, $B$ is the chirp bandwidth, $\alpha=B / T$ is the chirp rate, and $\beta$ is the carrier frequency, $\alpha, \beta$, $T$, and $B \in \mathbb{R}$. Choose the factorization $N=K L^{2}, L, K L \in$ $\mathbb{Z}^{+}$. The sequence

$$
x(n)=e_{L^{2}}\left(\frac{a n^{2}}{2}\right) e_{L}(b n), \quad a, b \in \mathbb{R}, a \neq 0,
$$

is called the discrete chirp, where $a=\alpha(T / K L)^{2}$ is the discrete chirp rate and $b=\beta(T / K L)$ is the discrete carrier frequency. To compactify expressions, we will use the following normalized chirp parameters, $\bar{a}=a K, \overline{\bar{a}}=a K^{2}$, and $\bar{b}=b K$.

We impose two conditions on the discrete chirp. First, to apply the Zak space techniques, we require that $x(n)$ be periodic with period $N$, that is,

$$
x(n+N)=x(n) .
$$

Since

$$
x(n+N)=x(n) e(\bar{a} n) e\left(\frac{\overline{\bar{a}} L^{2}}{2}+\bar{b} L\right)
$$

the condition (11) is satisfied if and only if

$$
\bar{a},(\overline{\bar{a}} L+2 \bar{b}) \frac{L}{2} \in \mathbb{Z} .
$$

Next, to facilitate Zak space processing, we impose the binary support constraint on the FZT of $x$ [27]. First, we need to define a few basic concepts. $X_{L}$ has a binary support iff

$$
\left|X_{L}(j, k)\right|= \begin{cases}\mathrm{A}, & (j, k) \in \operatorname{supp}\left(X_{L}\right) \subset \mathbb{N}_{L} \times \mathbb{N}_{K L}, \\ 0, & \text { else }\end{cases}
$$

where $A=\left\|X_{L}\right\|_{2} / \sqrt{\delta\left(X_{L}\right)} \in \mathbb{R}$ and $s\left(X_{L}\right)$ is the cardinality of the support of $X_{L}$. The binary support of $X_{L}$ is called minimal when $s\left(X_{L}\right)=K L$. We call a periodic discrete chirp having an FZT with a binary support a finite chirp.

Take $n=k+r K L, 0 \leq k<K L, 0 \leq r<L$. The chirp in (10) can then be expressed as

$$
x(k+r K L)=e_{N}\left(\frac{\bar{a} k^{2}}{2}+\bar{b} L k+\overline{\bar{a}} L k r\right) e\left(\frac{\overline{\bar{a}} r^{2}}{2}+\bar{b} r\right) .
$$


It follows from (2) that the FZT of $x$ has a binary support if and only if

$$
e\left(\frac{\overline{\bar{a}} r^{2}}{2}+\bar{b} r\right)=1
$$

or, equivalently,

$$
\frac{(\overline{\bar{a}}+2 \bar{b})}{2} \in \mathbb{Z} .
$$

Combining (13) and (17) leads to the Zak space condition for the finite chirp.

Theorem 1. The discrete chirp is $N$-periodic and has minimal support on the $L \times K L$ Zak transform lattice if and only if

$$
\bar{a}, \overline{\bar{a}},(\overline{\bar{a}}+2 \bar{b}) \frac{L}{2} \in \mathbb{Z} .
$$

The next result follows directly from substitution.

Theorem 2. Set $x_{k}:=e_{N}\left(\bar{a} k^{2} / 2+\bar{b} L k\right)$. Then, the $L \times K L F Z T$ of a finite chirp is

$$
X_{L}(j, k)= \begin{cases}L x_{k}, & \bar{a} k+j+\frac{\overline{\bar{a}} L}{2}+\bar{b} L \equiv 0(\bmod L) \\ 0, & \text { otherwise, }\end{cases}
$$

provided (18) is satisfied.

We call the congruence specifying the support of the FZT of a finite chirp an algebraic line. The availability of chirps with highly structured Zak space support suggests many applications. These include, among others, chirp rate and carrier frequency estimation, chirp detection, chirp denoising, chirp unmixing, reduced complexity computation of matched filters, and design of new sequence families with good correlation properties. This paper addresses the last two topics.

\section{FZT Tessellation}

Having introduced the FZT of a finite chirp, we can discuss issues related to FZT support more concretely. At the core of these issues is the close relationship between the DFT and the FZT. This relationship plays a key role in both theory and applications. In the latter case it leads, for example, to the reduced complexity realization of the Gerchberg-Papoulis algorithm [39]. In the former case it illuminates the structure of Zak space computations and permits an assessment of signal complexity. The purely computational aspect of this phenomenon has been remarked on in Section 2: the FZT arises formally from a sequence of DFTs performed on the decimated data set, when appropriately organized in a form of a $L \times K L$ array. The Zak space realization of a DFT pair provides a complementary view. Let $X_{L}$ be the FZT of $x$ and $Y_{K L}$ be the FZT of the DFT of $x$. Write the DFT of $x$

$$
\mathbf{x}(m)=\sum_{n=0}^{N-1} e_{N}(n m) x(n), \quad 0 \leq m<N,
$$

and set $n=k+r K L, m=j+s L, 0 \leq k, s<K L, 0 \leq r, j<L$, which leads to

$$
\mathbf{x}(j+s L)=\sum_{k=0}^{K L-1} \sum_{r=0}^{L-1} e_{N}[(k+r K L)(j+s L)] x(k+r K L) .
$$

After extracting the FZT of $x$, (21) can be rewritten as

$$
\mathbf{x}(j+s L)=\sum_{k=0}^{K L-1} e_{K L}(k s) e_{N}(j k) X_{L}(j, k) .
$$

Formula (22) is readily seen as the inverse FZT of $e_{N}(j k) X_{L}(j, k)$, that is,

Theorem 3.

$$
K L e_{N}(j k) X_{L}(j, k)=Y_{K L}(-k, j) .
$$

It follows from Theorem 3 that the FZTs of $x$ and $\mathbf{x}$ differ only by a ninety degree rotation and a complex factor multiplication.

Still, another way to view the relationship between DFT and FZT is by considering the range of values spanned by the FZT tesselation parameter, $L$. When $L=1$, then $K=N$ and the FZT is equal to the signal being transformed. When $L=N$, then $K=1 / N$, and the Zak transform is equal to the DFT of the signal. These two extreme cases are illustrated in Figure 1, together with the canonical, $L=\sqrt{N}$, choice of the FZT. The FZT in Figure 1 is supported at $L$ points on the algebraic line $\bar{a} k+j \equiv 0(\bmod L)$, while both the time signal and its DFT are nonzero everywhere [36]. The effect of chirp support compression in the Zak space has been investigated in depth in [27], and it was the main driving factor for transferring many chirp signal processing tasks to the Zak space $[25,37]$.

To consider the support of the FZT of a discrete chirp in greater generality, recall from [25] the following relaxation of (18).

Corollary 4. Take $\overline{\bar{a}}=n / d, n, d \in \mathbb{Z},(n, d)=1$. Then $s\left(X_{L}\right)=d K L$ iff

$$
\bar{a}, 2 \bar{b} L, \bar{b} L+\frac{n L}{2} \in \mathbb{Z} .
$$

Figure 1 shows the canonical choice for the Zak transform lattice parameter, $K=1$. Other choices of $K$ are possible, provided $N$ is sufficiently composite. A few of these choices are shown in Figure 2; the associated tessellations parameters are listed in Table 1. The tessellations $a=1 / 64, K=64$, $L=2, b=1 / 32$, and $a=64, K=1 / 64, L=128, b=2$ are not shown but they follow a similar pattern. The canonical lattice yields the sparsest representation of the finite chirp. As the FZT tessellation varies approaching either the time or the Fourier representation, the support of the transform becomes less sparse, and, as previously observed, becomes nonzero everywhere in the two limits. 

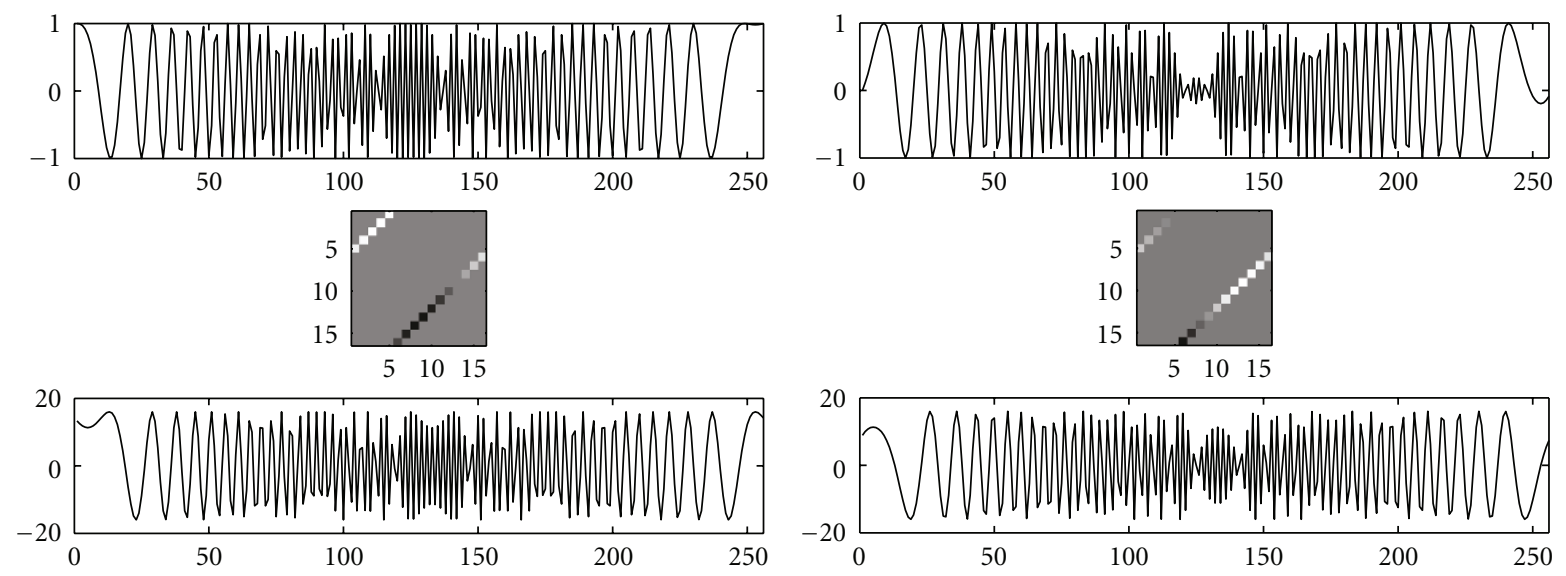

(a)

(b)

FIGURE 1: Real and imaginary parts of a chirp (top two plots), its FZT (middle plots), and its DFT (bottom plots). Chirp parameters: $a=1$, $K=1, L=16$, and $b=1 / 4$.

TABle 1: Parameters of chirps in Figure 2. $\bar{a}=1, \overline{\bar{a}}=K, N=256$, and $\bar{b} L=4$ for all chirps.

\begin{tabular}{lccccc}
\hline$a$ & $K$ & $L$ & $b$ & $2 \bar{b}$ & $d K L$ \\
\hline 16 & $1 / 16$ & 64 & 1 & $1 / 8$ & 64 \\
4 & $1 / 4$ & 32 & $1 / 2$ & $1 / 4$ & 32 \\
1 & 1 & 16 & $1 / 4$ & $1 / 2$ & 16 \\
$1 / 4$ & 4 & 8 & $1 / 8$ & 1 & 32 \\
$1 / 16$ & 16 & 4 & $1 / 16$ & 2 & 64 \\
\hline
\end{tabular}

Is the canonical representation always the sparsest? This is assured if the signal under consideration is a finite chirp or one of its generalizations discussed in Section 7, but it is not in many other cases. For example, for a chirp given by $N=256, \bar{a}=4$ and $\bar{b} L=4$, the most compact realization, $d K L=8$, is obtained for the choice of parameters $a=16$, $K=1 / 4, L=32$, and $b=1 / 2$.

\section{Zak Space Correlation}

The cyclic cross-correlation of two $N$-periodic polyphase sequences, $x$ and $y$, is given by

$$
z(n)=(y \circ x)_{n}:=\frac{1}{N} \sum_{m=0}^{N-1} y(m) x^{*}(m-n), \quad 0 \leq n<N
$$

where $m-n$ is taken modulo $N$. When $y=x$, the cyclic cross-correlation is called the cyclic autocorrelation. For computational efficiency the cyclic cross-correlation is often realized in the Fourier domain. Take $\mathbf{x}, \mathbf{y}$, and $\mathbf{z}$ to be the DFTs of $x, y$, and $z$, respectively. Then,

$$
\mathbf{z}=\frac{1}{N} \mathbf{y x}^{*}
$$

A sequence $x$ satisfies the perfect autocorrelation property if and only if

$$
(x \circ x)_{n}= \begin{cases}1, & n=0 \\ 0, & \text { otherwise }\end{cases}
$$

Furthermore, two distinct sequences, $x$ and $y$, satisfy the perfect cross-correlation property if and only if

$$
\left|(y \circ x)_{n}\right| \equiv N^{-1 / 2}
$$

Now, we can introduce the second main tool (after Theorem 2) for the study of polyphase sequences in Zak space. Take $X_{L}, Y_{L}$ and $Z_{L}$ to be the FZTs of $x, y$ and $z$ in (25), respectively. Write

$$
Z_{L}(j, k)=\sum_{r=0}^{L-1} z(k+r K L) e_{L}(r j)
$$

By (25), we have

$$
\begin{aligned}
& Z_{L}(j, k) \\
& =\frac{1}{N} \sum_{r=0}^{L-1} e_{L}(r j) \sum_{l=0}^{K L-1} \sum_{s=0}^{L-1} y(k+r K L) x^{*}(l+s K L-k-r K L) .
\end{aligned}
$$

Rearranging the RHS of (30), we have

$$
\begin{aligned}
& Z_{L}(j, k) \\
& =\frac{1}{N} \sum_{l=0}^{K L-1} \sum_{s=0}^{L-1} y(l+s K L) \sum_{r=0}^{L-1} e_{L}(r j) x^{*}((l-k)+(s-r) K L) .
\end{aligned}
$$



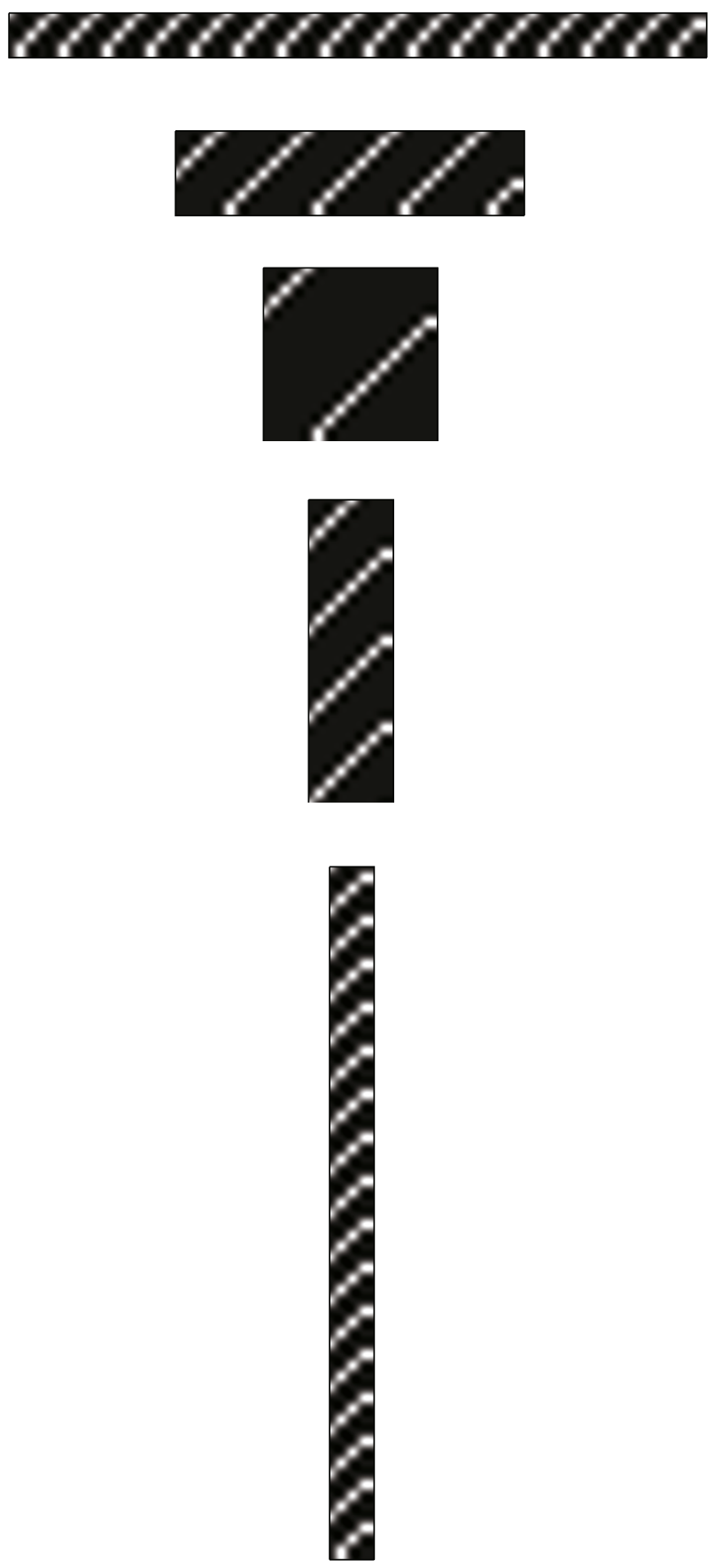

Figure 2: FZT magnitude of the finite chirp given by $\bar{a}=1, N=256$, and $\bar{b} L=4$ for $K=1 / 16,1 / 4,1,4$, and 16 (top to bottom).

Setting $p=s-r$ and again rearranging the terms on the RHS of (31) leads to

$$
\begin{aligned}
& Z_{L}(j, k) \\
& =\frac{1}{N} \sum_{l=0}^{K L-1} \sum_{s=0}^{L-1} e_{L}(s j) y(l+s K L) \sum_{p=0}^{L-1} e_{L}(-p j) x^{*}(l-k+p K L),
\end{aligned}
$$

which produces the Zak space correlation formula
Theorem 5.

$$
Z_{L}(j, k)=\frac{1}{N} \sum_{l=0}^{K L-1} Y_{L}(j, l) X_{L}^{*}(j, l-k) .
$$

The computation of $Z_{L}$, realized as a superposition of the inner products of $Y_{L}$ and $X_{L}$, and parameterized by a shift of $X_{L}$, is shown in Figure 3. Alternatively, the Zak space correlation can be viewed as a collection of $L K L$-point time 


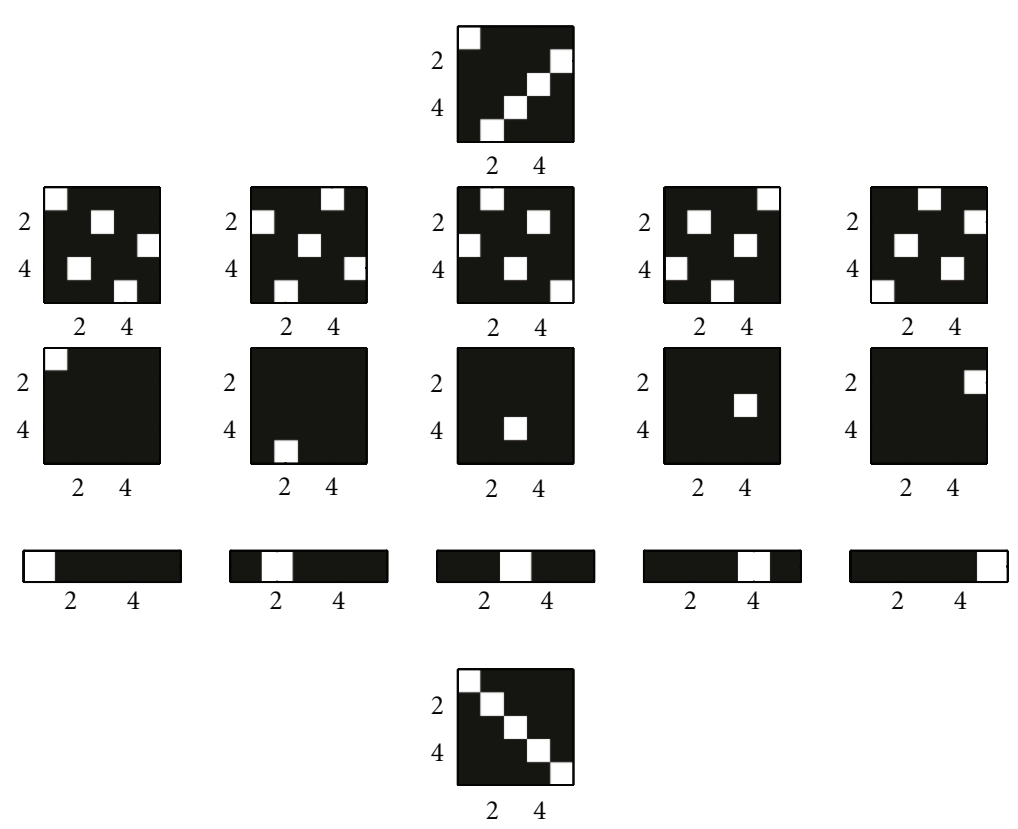

FIgure 3: Computation of the Zak space correlation of two finite chirps. To better illustrate the main idea, only the magnitude of FZTs is shown in the plots. First row: $Y_{L}$. Second row: cyclic shifts of $X_{L}$, from $X_{L}(j, k)$ to $X_{L}(j, k-4)$. Third row: pointwise products of $Y_{L}$ and cyclic shifts of $X_{L}$. Fourth row: sums of the pointswise products in $k$. Fifth row: concatenation of the vectors in the fourth row makes up the cross-correlation array $Z_{L}$.

domain cross-correlations performed on frequency slices of the $L \times K L$ Zak space signals, $X_{L}$ and $Y_{L}$. While the operations proceed identically for arbitrary $X_{L}$ and $Y_{L}$, the sparse support of Zak space chirps makes certain computations unnecessary, which suggests the possibility of adapting the correlation procedure to individual tasks and signals. This possibility will be explored in the next sections.

\section{Perfect Chirp Sets}

The main application of Theorem 5 discussed in this paper is the Zak space construction of PPSs. The construction includes families of finite chirps and families of certain more general sequences that are related to chirps. The next several results specify perfect correlation conditions for sets of finite chirps. This is followed by a construction of a perfect sequence set. We begin with a statement of the perfect autocorrelation condition for finite chirps.

Theorem 6. A finite chirp satisfies the perfect autocorrelation property if and only if

$$
(\bar{a}, K L)=1
$$

A finite chirp satisfying condition (34) is called a bat chirp. In the following discussion we will identify a collection of bat chirps that additionally satisfy the perfect crosscorrelation property. We focus on the case $K=1$, but a more general construction is easily available. The first result provides an explicit description of the FZT of crosscorrelation of bat chirps. This is a simplified version of result described in [25].
Theorem 7. Take $X_{L}(j, k)$ to be the FZT of a bat chirp, and consider the set

$$
\begin{aligned}
& \mathcal{B}_{L} \\
& =\left\{X_{L}(j, k) \mid K=1, L \text { an odd prime, } 1 \leq \bar{a}<L, 2 \bar{b} \in \mathbb{Z}\right\}
\end{aligned}
$$

Take any two chirps $y$ and $x$, with the chirp rates $\bar{a}_{1}$ and $\bar{a}_{2}, \bar{a}_{1} \not \equiv \bar{a}_{2}(\bmod L)$, and the carrier frequencies $\bar{b}_{1}$ and $\bar{b}_{2}$. Suppose that the Zak transforms of $y$ and $x, Y_{L}(j, k)$ and $X_{L}(j, k)$, respectively, are in $\mathcal{B}_{L}$. Then the Zak transform of the cross-correlation of $y$ and $x$ is given by

$$
Z_{L}(j, k)= \begin{cases}z_{k}, & {\left[\frac{\bar{a}_{1} \bar{a}_{2}}{\bar{a}_{2}-\bar{a}_{1}}\right]_{L} k+j \equiv 0(\bmod L),} \\ 0, & \text { otherwise, }\end{cases}
$$

where

$$
\begin{gathered}
z_{k}=e_{N}\left(\frac{\bar{a}_{3} k^{2}}{2}\right) e_{L}\left(\bar{b}_{3} k\right), \\
\bar{a}_{3}=\bar{a}_{1}\left[\frac{\bar{a}_{2}}{\bar{a}_{2}-\bar{a}_{1}}\right]_{L}^{2}-\bar{a}_{2}\left[\frac{\bar{a}_{1}}{\bar{a}_{2}-\bar{a}_{1}}\right]_{L}^{2}, \\
\bar{b}_{3}=\bar{b}_{2}+\left(\bar{b}_{1}-\bar{b}_{2}\right)\left[\frac{\bar{a}_{2}}{\bar{a}_{2}-\bar{a}_{1}}\right]_{L},
\end{gathered}
$$

and $[a]_{L}$ denotes $a(\bmod L)$.

The next result states the perfect cross-correlation condition for bat chirps. 

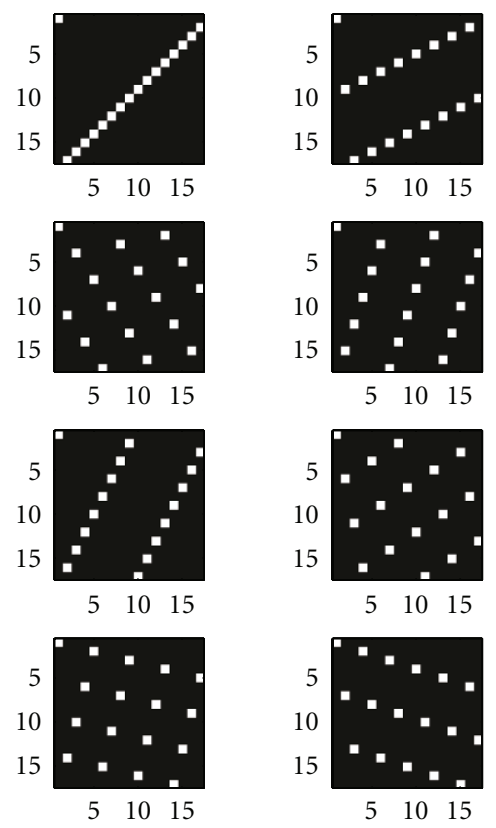

Figure 4: FZT magnitude of PCS sequences for $L=17 \cdot \bar{b}=1$ when $\bar{a}$ is even, and $\bar{b}=1 / 2$ otherwise.

Corollary 8. Any two bat chirps with $\bar{a}_{1} \neq \bar{a}_{2}$ whose FZTs are in $\mathscr{B}_{L}$ satisfy the perfect cross-correlation condition.

We call the set $\mathscr{B}_{L}$ the perfect chirp set (PCS). The PCS contains $L-1$ sequences, parameterized by the values of the chirp rate. An example of a PCS for $L=17$ is shown in Figure 4. The family of PCSs is, essentially, identical with the family of Zadoff-Chu sequences, when the length of the sequence is a square of an odd prime. However, the Zak space construction, unlike the Zadoff-Chu construction, is not limited to chirps. This is elucidated in the next section.

Remark 9. The constraints: $K=1, L$ are an odd prime, and $1 \leq \bar{a}<L$ in (35) can be relaxed in some cases, leading to the construction of smaller PCSs. For example, we can lift the requirement that $L$ be an odd prime, provided the condition

$$
\left(\bar{a}-\bar{a}^{\prime}, K L\right)=1
$$

is satisfied for every pair of chirps in the set

$$
\mathcal{B}_{L}^{\prime}=\left\{X_{L}(j, k) \mid K=1,1 \leq \bar{a}<L,(\overline{\bar{a}}+2 \bar{b}) \frac{L}{2} \in \mathbb{Z}\right\} .
$$

We illustrate this effect in the next two examples.

Example 10. Take $L=16$. The only chirps in the set

$$
S_{1}=\left\{X_{L} \mid K=1, L=16,1 \leq \bar{a}<L\right\}
$$

that satisfy (34) are chirps with odd valued chirp rates (Figure 5). Moreover, since all differences of chirp rates $\bar{a}-\bar{a}^{\prime}$ of chirps in the set share a common factor with $L$, no subset of $S_{1}$ is a PCS.
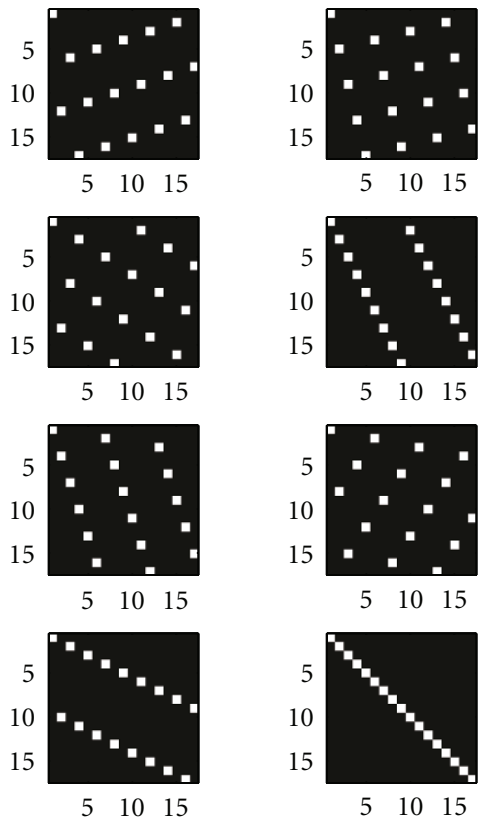

Example 11. Take $L=15$. The only chirps in the set

$$
S_{2}=\left\{X_{L} \mid K=1, L=15,1 \leq \bar{a}<L\right\}
$$

that satisfy the condition (34) are chirps with chirp rates $\bar{a}=1,2,4,7,8,11,13$, and 14 . Twelve subsets of $\mathrm{S}_{2}$ form two-element PCSs. The associated pairs of chirp rates are: $(1,2),(1,8),(1,14),(2,4),(2,13),(4,8),(4,11),(7,8)$, $(7,11),(7,14),(11,13)$, and $(13,14)$.

Remark 12. It is useful to note that while no subset of $S_{1}$ is a PCS, pairs of chirps with odd-valued chirp rates that are subsets of $S_{1}$ have a two-valued cross-correlation, equal either to zero or to $\sqrt{\left(\bar{a}_{1}-\bar{a}_{2}, L\right)} / L$. For example, the pairs of chirps $(1,3)$ and $(1,5)$ have cross-correlations with the maximum values of $\sqrt{2} / L$ and $2 / L$, respectively.

\section{Generalizations}

In the previous section, we introduced the PCS. Here, we describe the two principal relaxations of the PCS to a perfect sequence set (PSS). Sequences contained in PSS satisfy, like sequences contained in PCS, the perfect correlation properties (27) and (28), but they are not necessarily chirps.

\subsection{Relaxation of the Modulation Constraint}

Corollary 13. Let $X_{L}(j, k)$ be an arbitrary $L \times L$ complexvalued array, such that

$$
\left|X_{L}(j, k)\right|= \begin{cases}L, & \bar{a} k+j \equiv 0(\bmod L), \\ 0, & \text { otherwise. }\end{cases}
$$


Then, the set of inverse FZTs of elements of the set

$$
\S=\left\{X_{L}(j, k) \mid K=1, \quad L \text { an odd prime, } 1 \leq \bar{a}<L\right\}
$$

is a PSS.

Example 14. Let

$$
X_{L}(j, k)= \begin{cases}L e_{N}(p(k)), & \bar{a} k+j \equiv 0(\bmod L), \\ 0, & \text { otherwise }\end{cases}
$$

where $p(k)$ is a polynomial in $k$. Then, the set

$$
\mathrm{FZT}^{-1}\left\{X_{L}(j, k) \mid K=1, L \text { an odd prime, } 1 \leq \bar{a}<L\right\}
$$

is a PSS.

Example 15. Consider two chirps as in Example 10, but each modulated by a distinct complex factor. It can be shown that while the maximum cross-correlation sidelobe value is still $\sqrt{\left(\bar{a}_{1}-\bar{a}_{2}, L\right)} / L$, the cross-correlation is no longer twovalued.

7.2. Relaxation of the Support Constraint. Corollary 13 suggests that a PCS can be extended in a straightforward fashion to the set of generalized Frank sequences. A further generalization of $\&$ can be obtained by observing that the Zak space support of a perfect sequence does not need to be restricted to an algebraic line. In fact, any unimodular sequence that has a support on the Zak transform lattice at indexes specified by an appropriately chosen permutation sequence is a perfect sequence. This statement is made precise in [25], where it is shown that the set of all perfect autocorrelation sequences associated with the set $\mathcal{B}_{L}^{\prime}$ can be factored into $(L-2)$ ! PSSs. The construction is outlined in the next example.

Example 16. Fix $L=5$. The PSS sequences are given by lists of indices $j$ (except for $j=0$, for which $k=0$ ), ordered in $k$, of the nonzero values of the associated $L \times L$ FZTs
(1) $(1,2,3,4),(2,4,1,3),(3,1,4,2),(4,3,2,1)$
(2) $(1,2,4,3),(2,4,3,1),(3,1,2,4),(4,3,1,2)$
(3) $(1,3,2,4),(2,1,4,3),(3,4,1,2),(4,2,3,1)$
(4) $(1,3,4,2),(2,1,3,4),(3,4,2,1),(4,2,1,3)$
(5) $(1,4,2,3),(2,3,4,1),(3,2,1,4),(4,1,3,2)$
(6) $(1,4,3,2),(2,3,1,4),(3,2,4,1),(4,1,2,3)$.

The collection of PSSs forms a partition of the sets of all perfect autocorrelation sequences. The first PSS in the partition is the set of generalized Frank sequences. The remaining PSSs are formed by appropriate permutations of sequences in the first PSS [25]. The construction of a partition of the set of all perfect autocorrelation sequences into PSSs proceeds as follows.
(1) Start with the sequence $(1,2, \ldots, L-1)$, and apply the mapping $k \mapsto-\bar{a} k(\bmod L), 1 \leq \bar{a}<L-1$ to each of its elements.

(2) Generate the " $j$ " sequences by reordering the sequence $(1,2, \ldots, L-1)$ according to the index sequences obtained in the previous stage. This yields the first PSS.

(3) For each sequence in the first PSS, compute $(L-2)$ ! permutations of its last $L-2$ elements. Each permutation generates a new PSS. with the remaining element being fixed. There are $(L-2)$ ! such sequences.

The construction can be described more formally using the language of group theory. The main stage of the construction is the coset decomposition of a certain permutation group [25].

\section{Matched Filter}

The most direct application of the cross-correlation formula (33) is the Zak space implementation of the matched filter. Matched filter processing is used in many radar, sonar, and communications tasks [10, 20, 26, 31, 33, 43, 44].

Set $x$ to be the probing signal and $y$ the received or echo signal. Suppose that $y$ is delayed by $s \in \mathbb{Z}$ and attenuated by $a \in \mathbb{R}^{+}$replica of $x$, that is,

$$
y(n)=a x(n-s), s=p+q L, 0 \leq p, q<L .
$$

The matched filter for $y$ is given by the cross-correlation

$$
z(n)=\frac{1}{N} \sum_{m=0}^{N-1} a x(m-s) x^{*}(m-n), \quad 0 \leq n<N
$$

Suppose that $x$ is a bat chirp. Then, it follows from (33) that the Zak transform of $z$ is

$$
Z_{L}(j, k)= \begin{cases}a e_{L}(j q), & k=p \\ 0, & \text { otherwise. }\end{cases}
$$

In general, when $y$ is a sum of delayed and attenuated replicas of $x$, that is,

$$
y(n)=\sum_{d=0}^{D-1} a_{d} x\left(n-s_{d}\right), \quad s_{d}=p_{d}+q_{d} L, 0 \leq p_{d}, q_{d}<L,
$$

then the Zak space matched filter can be viewed as a sum, over $d$, of a sequence of individual matched filters of the form

$$
Z_{L}^{(d)}(j, k)= \begin{cases}a_{d} e_{L}\left(j q_{d}\right), & k=p_{d} \\ 0, & \text { otherwise. }\end{cases}
$$

This view is strictly formal, of course; it is far more efficient to compute the Zak transform of a sum of signals than the sum of the respective Zak transforms. 
The reason for considering a matched filter in the Zak space is that the sparse and highly structured Zak space support of pulse compression signals avails a radically different view of the cross-correlation task. The full advantage of this view was taken in the sequence design work described in previous sections. In the case of the matched filter, the benefit is more modest but still significant. The advantage is twofold.

First, in contrast with either time or frequency space representations, the Zak space representation of echo signals preserves the separateness of supports of distinct replicas of the probing signal. This is true of all cases, except for replicas whose delay times differ by a multiple of $L$. As each replica is an algebraic line on the FZT lattice, by the shift property of the FZT, differently delayed replicas are parallel lines. In effect, the Zak space replicas can be better distinguished than either the time or the frequency space replicas, even in the presence of noise, when the Zak space lines become degraded. Figures 6, 7, and 8, showing an example of a matched filter realized in the Zak space, succinctly illustrate this point. The geometric aspect of Zak space processing is also present in the Zak transform of the matched filter, $Z_{L}$. A match of a probing signal and a replica in the Zak space is a horizontal line on the FZT lattice (Figure 8 ). If a replica is delayed by more than $L$, this line is modulated by the factor $e_{L}(j q)$. If a replica is delayed by less than $L$, all points on the line have constant magnitude with zero imaginary part. These geometric effects can be taken advantage of by combining classical signal estimation procedures with various image processing techniques. Some approaches toward that end have been suggested in [37].

Second, the Zak space implementation of the matched filter has a computational complexity advantage over the standard Fourier space realization. The Fourier space implementation of the matched filter requires the computation of the DFT of the echo, $N$ pointwise multiplication of DFTs of the probing and received signals, and an inverse DFT of the product of the two DFTs. Jointly these tasks require $\mathrm{N}(1+$ $\left.2 \log _{2} N\right)$ multiplications. The Zak space implementation of the matched filter requires the computation of the FZT of the echo, $N$ multiplications for realization of the Zak space crosscorrelation, and an inverse FZT of the Zak space correlation. Jointly these tasks require $N\left(1+2 \log _{2} L\right)$ multiplications. In effect, the Zak space implementation of the matched filter achieves nearly 50\% reduction in the computational cost of the Fourier space realization.

\section{Open Problems}

The Zak transform methods avail a powerful new framework for the design and analysis of sequences with good correlation properties. The key feature of this framework is the two-dimensional time-frequency analysis space that is closely coupled with the Fourier space. This setting permits characterization of PPSs in terms of two separable operations: modulation and permutation. These operations can be conveniently related to the individual steps of the Zak space correlation. Prior investigations utilizing this framework led to reframing of some well-known sequence design results in the Zak transform language and to the design of new sequence sets $[25,37]$. While these results are useful, they suggest further inquiries into the fundamental structure of the Zak space. Among the principal tasks in this area are:

(1) exact specification of the class of PPSs amenable to the Zak transform methods,

(2) characterization of the abstract algebraic properties of certain families of PPSs; this task includes extending the results on closure of PCS under DFT and correlation, postulated in [18] and given in [25],

(3) construction of design guidelines for embedding additional properties, such as acyclic correlation properties, sub-optimal cyclic correlation properties, and doppler immunity, into PPSs,

(4) investigation of higher dimensional spaces as potential settings for PPS design,

(5) investigation of potential new constructions of binary and generalized Barker codes.

The first of these problems is particularly important. In [25], it was shown that the only unimodal FZT associated with a PSS is an FZT supported on an algebraic line. We will make the following claim.

Conjecture 17. Every PPS is associated with an FZT supported on an algebraic line.

If Conjecture 17 is true then the PPS design can be completely transferred to the Zak space. This change of design settings might inspire many new investigations. For example, one of the outstanding problems in sequence design is verification of existence of PPSs for various sequence sizes [12]. In a recent work, Mow proposed that the number of PPSs, whose length is a square of a prime is greater than or equal to $L ! N^{L}$ [15]. If conjecture 1 is true, then it can be shown that the Mow bound is tight. The argument is based on the observation that there are $L$ ! possible choices for an algebraic line (including cyclic shifts) on a square FZT lattice, and that for each algebraic line each of the $L$ nonzero values of the FZT of a PPS can assume one of exactly $N$ values (the $N$ th roots of unity). The number of PPSs can be slightly refined when a different accounting method is used. For example, after removing the sequences that vary only by a cyclic shift $(N)$ or a constant factor multiplication $(N)$, the number of PPSs is reduced to $L ! N^{L-2}$. We call these PPSs the unique PPSs (UPPSs).

Example 18. Take $L=2$. The number of UPPSs is $L ! N^{L-2}=$ $L !=2$. There is only one shift-invariant permutation of $X_{2}(j, k)$ that can be associated with a UPPS, given by $X_{2}(j, k) \neq 0$ for $j=k$ and zero otherwise. Set $X_{2}(0,0)=$ $e_{2}(0)=1$ and $X_{2}(1,1) \in\left\{e_{4}(0), e_{4}(1), e_{4}(2), e_{4}(3)\right\}=$ $\{1, i,-1,-i\}$. The inverse FZT in these four cases yields the sequences $(1,1,1,-1),(1, i, 1,-i),(1,-1,1,1)$, and $(1,-i, 1, i)$. Note that for brevity, we skip the scaling factor, $L^{-1}$, here and in the next example. 

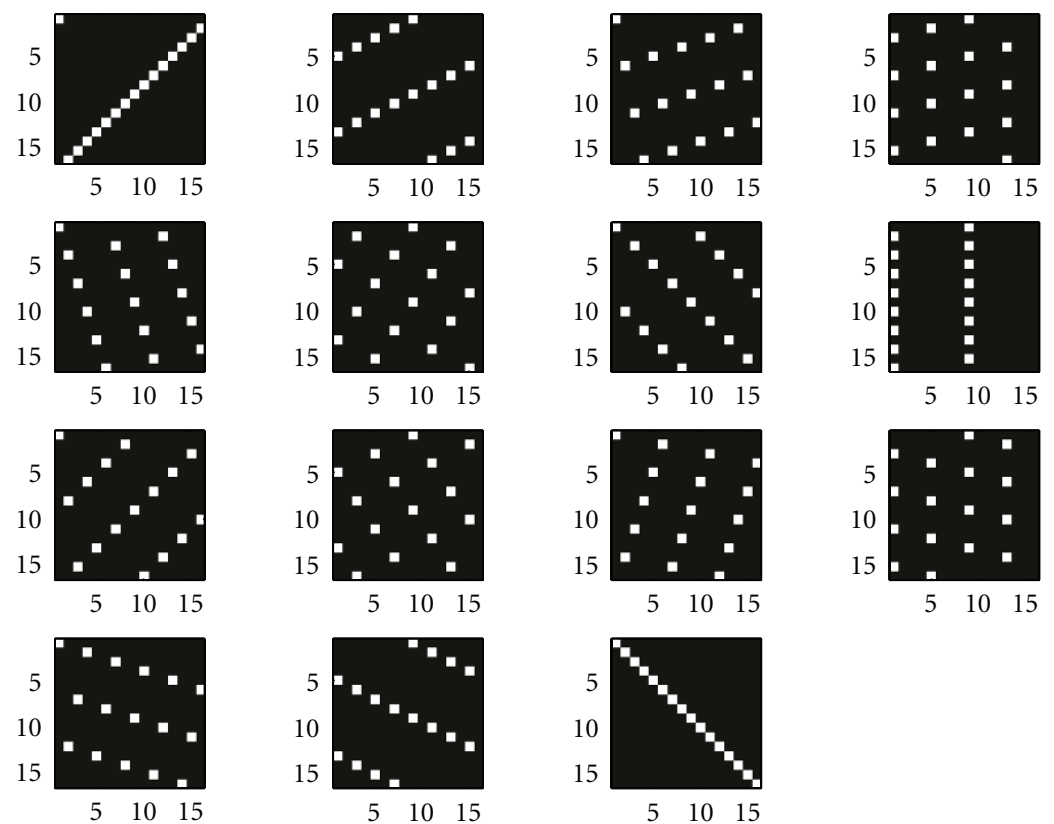

Figure 5: FZT magnitude of sequences in $S_{1}$ (Example 10) in lexicographical order according to the value of $\bar{a}$. Only sequences with odd valued $\bar{a}$, that is, $\bar{a}=1,3,5,7,9,11,13,15$ satisfy the perfect autocorrelation condition $(34) . \bar{b}=1 / 2$ for all $\bar{a}$.

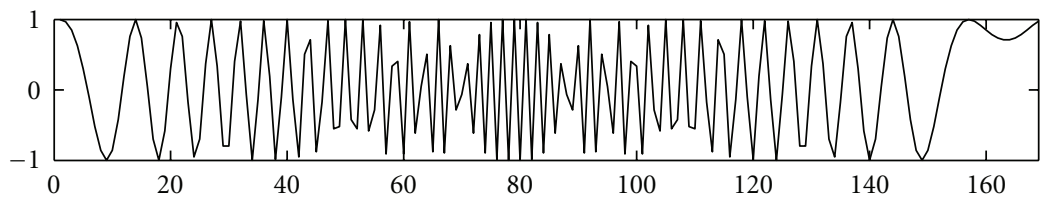

(a)

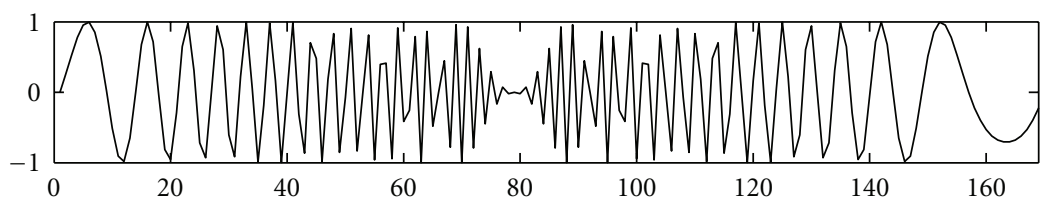

(b)

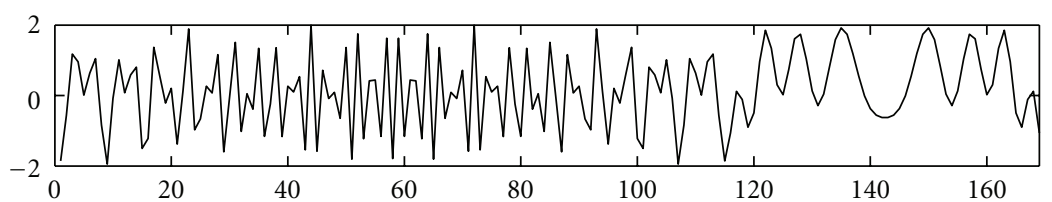

(c)

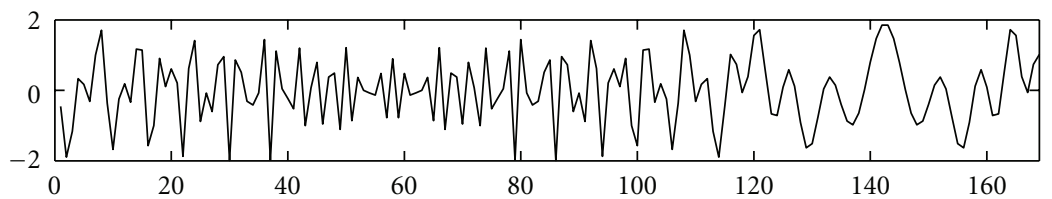

(d)

Figure 6: (a)-(d): real and imaginary parts of the chirp $x$, and real and imaginary parts of the echo $y$, where $y$ is the sum of replicas of $x$ delayed by 9 and $7+2 L$. Chirp parameters: $\bar{a}=1, N=169$, and $\bar{b} L=13 / 2$. 


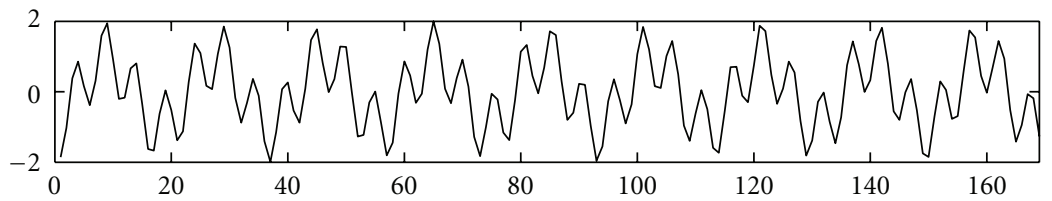

(a)

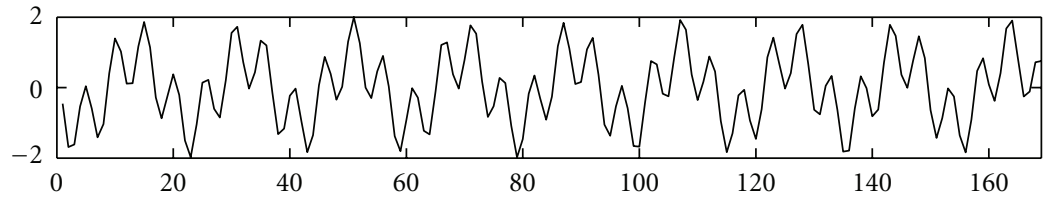

(b)

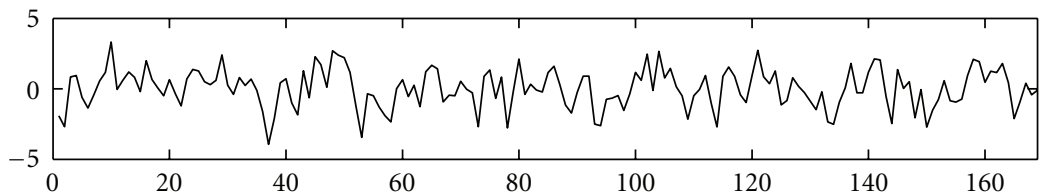

(c)

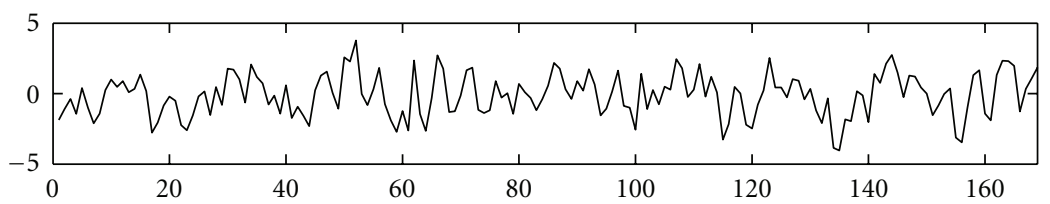

(d)

Figure 7: (a)-(d): real and imaginary parts of Fourier space cross-correlation of signals from Figure 6. The upper two plots show the noise-free case and the lower two plots show the case when signal is contaminated by $0 \mathrm{~dB}$ noise.
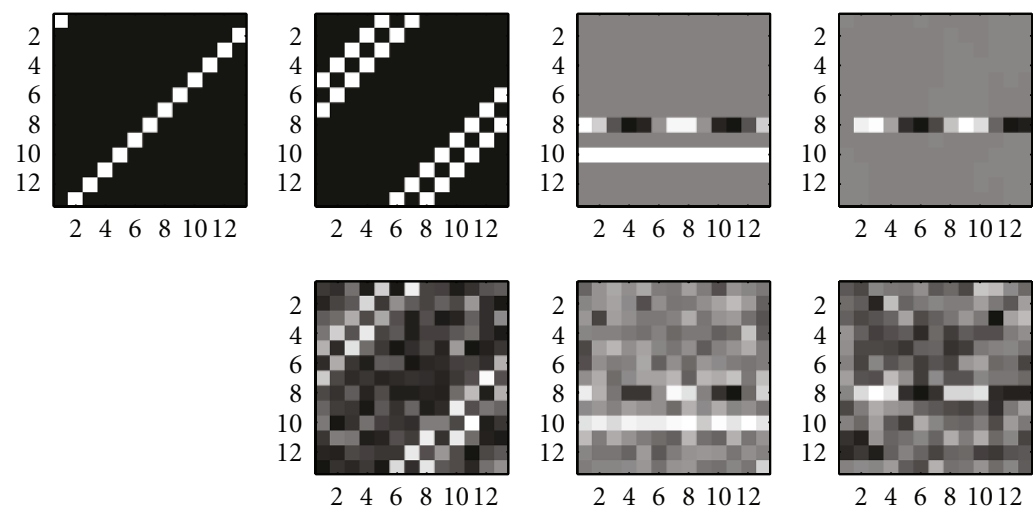

(a)

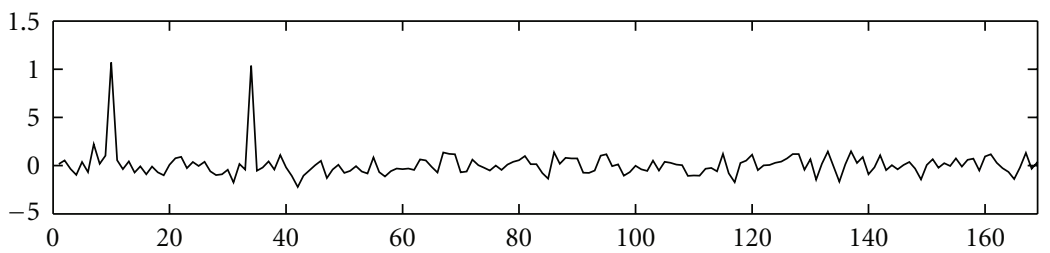

(b)

FIGURE 8: Zak space realization of matched filter of signals from Figure 6. Lexicographically: FZT magnitude of the chirps $x$ and $y$, real and imaginary parts of Zak space cross-correlation in no noise, FZT magnitude of the echo signal, $y$, in $0 \mathrm{~dB}$ noise, real and imaginary parts of Zak space cross-correlation in $0 \mathrm{~dB}$ noise, and the inverse Zak transform of Zak space cross-correlation in $0 \mathrm{~dB}$ noise. 
Example 19. Take $L=3$. The number of UPPSs is $L ! N^{L-2}=$ $L ! N=6 * 9=54$. Suppose that $X_{3}(j, k) \neq 0$ when $j=k$ and zero otherwise. Then,

$$
\begin{aligned}
& x(0+r L)=X_{3}(1,1) e_{3}(0) \\
& x(1+r L)=X_{3}(2,2) e_{3}(r) \\
& x(2+r L)=X_{3}(3,3) e_{3}(2 r)
\end{aligned}
$$

Fix $X_{3}(0,0)=1$ and let $X_{3}(1,1) \in\left\{e_{9}(0), \ldots, e_{9}(2)\right\}$ and

\begin{tabular}{|c|c|c|c|c|c|c|c|c|}
\hline & & & & & & 0 & 56 & \\
\hline & & & & & & & & \\
\hline & & & & 0 & & & & \\
\hline & 030 & 0 & & 040 & & 3 & & \\
\hline & 03 & 06 & $=$ & 041 & & 4 & & \\
\hline & 03 & 06 & $(0$ & 04 & 07 & 025 & 052 & \\
\hline & 03 & 06 & $(0$ & 04 & & (026 & & \\
\hline & & & (017) & & 07 & 102 & & \\
\hline & & & 10 & & & 02 & & \\
\hline
\end{tabular}
$X_{3}(2,2) \in\left\{e_{9}(0), \ldots, e_{9}(8)\right\}$. Then, half of the UPPSs are given by

where $\left(n_{0}, \ldots, n_{L^{2}-1}\right), n_{i} \in \mathbb{Z} \bmod L^{2}$ denotes $\left(e_{L^{2}}\left(n_{0}\right), \ldots, e_{L^{2}}\left(n_{L^{2}-1}\right)\right)$. Taking $X_{3}(j, k)=1$ when $j=2 k$ and zero otherwise produces the remaining UPPSs (e.g., (000 063 036), etc.).

We will comment on other open problems only briefly. Problems 2 and 3 are related. As explained in [25], PPS sets can be associated with cyclic groups. Can more general structures be defined that combine certain cosets of these groups so that while the perfect correlation property is moderately relaxed, the sequence set size increases? Can these structures be parameterized by the values of both chirp rate and carrier frequency in the case of PCS? Can a similar sequence design formalism be developed for the acyclic correlation? The FZT of the acyclic correlation is closely coupled with the expression for the FZT of a zeropadded chirp. This topic was discussed in [37]. Among other things, it was shown that the Zak space correlation of chirps can be used in sensing applications to distinguish between closely spaced radar echoes. It remains to be shown how chirp modulation may affect these computations.

It was stated in the introduction that chirps and chirp-like sequences are intrinsically high dimensional. Subsequently, we analyzed these sequences in the twodimensional Zak space. Are other multidimensional constructions, including higher dimensional Zak transforms, potentially advantageous, at least for some factorizations of the sequence length? It follows directly from the Zak space cross-correlation formula that the Zak space analysis cannot be improved directly, as the $L$-point support of PPS is the smallest that can be achieved with the Zak space methods.
Can PPSs be compactified to a fewer than $L$ nonzero values using other methods?

A question of significant theoretical and practical interest, which extends beyond the focus on PPSs, concerns the exact relationship between the FZT and the discrete fractional Fourier transform [34]. Both of these transforms are closely related to the DFT and both are linked to chirps, yet no major work has so far compared these two important signal-analytic tools. Indeed, the field of Zak space analysis appears to us as the field of sequence design did some fifty years ago to Woodward [10]: while the results discovered are clearly useful over a wide range of applications, much of the underlying theory still remains hidden.

\section{Acknowledgment}

The author would like to thank Julie DelVecchio Savage for support of this work.

\section{References}

[1] S. W. Golomb and G. Gong, Signal Design for Good Correlation, Cambridge University Press, Cambridge, UK, 2005.

[2] P. Fan and M. Darnell, Sequence Design for Communications Applications, John Wiley \& Sons, New York, NY, USA, 1996.

[3] T. Helleseth and P. V. Kumar, "Sequences with low correlation," in Handbook of Coding Theory, V. S. Pless and W. C. Huffman, Eds., pp. 1765-1854, Elsevier, New York, NY, USA, 1998.

[4] C. F. Gauss, Disquisitiones Arithmeticae, Springer, New York, NY, USA, 1986, (first published 1801).

[5] S. Sidon, "Ein Satz über trigonometrische Polynome und seine Anwendung in der Theorie der Fourier-Reihen," Mathematische Annalen, vol. 106, no. 1, pp. 536-539, 1932.

[6] P. Erdös and P. Turan, "On a problem of Sidon in additive number theory," Journal of the London Mathematical Society, vol. 3, no. 270, pp. 212-215, 1941.

[7] J. E. Littlewood, Some Problems in Real and Complex Analysis, D C Heath, Lexington, Mass, USA, 1968.

[8] M. R. Schroeder, Number Theory in Science and Communication, Springer, Berlin, Germany, 1984.

[9] P. Borwein, Computational Excursions in Analysis and Number Theory, Springer, New York, NY, USA, 2000.

[10] P. M. Woodward, Probability and Information Theory with Applications to Radar, McGraw-Hill, New York, NY, USA, 1953.

[11] J. R. Klauder, A. C. Price, S. Darlington, and W. J. Albersheim, "The theory and design of chirp radars," Bell System Technical Journal, vol. 39, pp. 745-808, 1960.

[12] G. Björck and B. Saffari, "New classes of finite unimodular sequences with unimodular Fourier transforms. Circulant Hadamard matrices with complex entries," Comptes Rendus de l'Académie des Sciences. Série 1, Mathématique, vol. 320, pp. 319-324, 1995.

[13] D. C. Chu, "Polyphase codes with good periodic correlation properties," IEEE Transactions on Information Theory, vol. 18, no. 4, pp. 531-532, 1972.

[14] R. L. Frank and S. A. Zadoff, "Phase shift pulse codes with good periodic correlation properties," IRE Transactions on Information Theory, vol. 8, pp. 381-382, 1962. 
[15] W. H. Mow, Sequence Design for Spread Spectrum, The Chinese University Press, Hong Kong, 1995.

[16] A. M. Odlyzko, "Construction of invertible sequences for multipath estimation," in Communications and Cryptography: Two Sides of One Tapestry, R. E. Blahut, D. J. Costello Jr., U. Maurer, and T. Mittelholzer, Eds., pp. 323-331, Kluwer Academic Publishers, Dordrecht, The Netherlands, 1994.

[17] D. V. Sarwate, "Bounds on crosscorrelation and autocorrelation of sequences," IEEE Transactions on Information Theory, vol. 25, no. 6, pp. 720-724, 1979.

[18] R. A. Scholtz and L. R. Welch, "Group characters: sequences with good correlation properties," IEEE Transactions on Information Theory, vol. 24, no. 5, pp. 537-545, 1978.

[19] N. Suehiro and M. Hatori, "Modulatable orthogonal sequences and their application to SSMA systems," IEEE Transactions on Information Theory, vol. 34, no. 1, pp. 93-100, 1988.

[20] J. Li and P. Stoica, Eds., MIMO Radar Signal Processing, WileyIEEE Press, New York, NY, USA, 2008.

[21] J. Tan and G. L. Stüber, "Multicarrier spread spectrum system with constant envelope: antijamming, jamming estimation, multiuser access," IEEE Transactions on Wireless Communications, vol. 4, no. 4, pp. 1527-1538, 2005.

[22] P. E. Pace, Detecting and Classifying Low Probability of Intercept Radar, Artech House, London, UK, 2009.

[23] S. Golomb, M. G. Parker, and A. Pott, Eds., "Sequences and Their Applications_-SETA 2008," Springer, New York, NY, USA, 2008.

[24] J. J. Benedetto, I. Konstantinidis, and M. Rangaswamy, "Phasecoded waveforms and their design: the role of the ambiguity function," IEEE Signal Processing Magazine, vol. 26, no. 1, pp. 22-31, 2009.

[25] A. K. Brodzik and R. Tolimieri, "Bat chirps with good properties: Zak space construction of perfect polyphase sequences," IEEE Transactions on Information Theory, vol. 55, no. 4, pp. 1804-1814, 2009.

[26] A. W. Rihaczek, Principles of High-Resolution Radar, Peninsula Publishing, Los Altos, Calif, USA, 1985.

[27] A. K. Brodzik, "Characterization of Zak space support of a discrete chirp," IEEE Transactions on Information Theory, vol. 53, no. 6, pp. 2190-2203, 2007.

[28] R. M. Lerner, "Representation of signals," in Lectures on Communication System Theory, E. J. Baghdady, Ed., pp. 203 242, McGraw-Hill, New York, NY, USA, 1961.

[29] P. Flandrin, Time-Frequency/Time-Scale Analysis, Academic Press, San Diego, Calif, USA, 1998.

[30] A. J. E. M. Janssen, "On the locus and spread of pseudodensity functions in the time-frequency plane," Philips Journal of Research, vol. 37, no. 3, pp. 79-110, 1982.

[31] R. A. Altes, "Detection, estimation, and classification with spectrograms," Journal of the Acoustical Society of America, vol. 67 , no. 4, pp. 1232-1246, 1980.

[32] M. Morvidone and B. Torresani, "Time-scale approach for chirp detection," International Journal of Wavelets, Multiresolution Analysis and Information Processing, vol. 1, no. 1, pp. 19-49, 2003.

[33] O. Akay and G. F. Boudreaux-Bartels, "Fractional convolution and correlation via operator methods and an application to detection of linear FM signals," IEEE Transactions on Signal Processing, vol. 49, no. 5, pp. 979-993, 2001.

[34] H. M. Ozaktas, Z. Zalevsky, and M. A. Kutay, The Fractional Fourier Transform, Wiley, New York, NY, USA, 2001.
[35] M. An, A. K. Brodzik, I. Gertner, and R. Tolimieri, "Weylheisenberg systems and the finite Zak transform," in Signal and Image Representation in Combined Spaces, Y. Zeevi and R. Coifman, Eds., pp. 3-22, Academic Press, San Diego, Calif, USA, 1998.

[36] A. Brodzik, "On the Fourier transform of finite chirps," IEEE Signal Processing Letters, vol. 13, no. 9, pp. 541-544, 2006.

[37] M. An, A. K. Brodzik, and R. Tolimieri, Ideal Sequence Design in Time-Frequency Space, Applications in Radar, Sonar and Communication Systems, Springer, Boston, Mass, USA, 2008.

[38] J. Zak, "Finite translations in solid-state physics," Physical Review Letters, vol. 19, no. 24, pp. 1385-1387, 1967.

[39] A. K. Brodzik and R. Tolimieri, "Extrapolation of band-limited signals and the finite Zak transform," Signal Processing, vol. 80, no. 3, pp. 413-423, 2000.

[40] I. Daubechies, S. Jaffard, and J.-L. Journe, "A simple Wilson orthonormal basis with exponential decay," SIAM Journal on Mathematical Analysis, vol. 22, no. 2, pp. 554-573, 1991.

[41] A. J. E. M. Janssen, "The Zak transform: a signal transform for sampled time-continuous signals," Philips Journal of Research, vol. 43, no. 1, pp. 23-69, 1988.

[42] K. Gröchenig, Foundations of Time-Frequency Analysis, Birkhäuser, Boston, Mass, USA, 2000.

[43] N. Levanon and E. Mozeson, Radar Signals, Wiley, New York, NY, USA, 2004.

[44] D. E. Vakman, Sophisticated Signals and the Uncertainty Principle in Radar, Springer, New York, NY, USA, 1968. 